\title{
Conformity and truthful voting under different voting rules
}

Bernardo Moreno, María del Pino Ramos-Sosa, and Ismael Rodríguez-Lara

\section{ThE PAPERS}

DPTO. TEORÍA E HISTORIA ECONÓMICA WORKING PAPER SERIES UNIVERSIDAD DE GRANADA 


\section{Conformity and truthful voting under different voting rules}

Bernardo Moreno, María del Pino Ramos-Sosa, and Ismael Rodríguez-Lara $19 / 04$

\section{SUGGESTED CITATION}

Bernardo Moreno, María del Pino Ramos-Sosa, and Ismael Rodríguez-Lara (2019). Conformity and truthful voting under different voting rules. ThE Papers, Department of Economic Theory and Economic History of Universidad de Granada. 19/04. 


\title{
Conformity and truthful voting under different voting rules
}

\author{
Bernardo Moreno, ${ }^{1}$ Maria del Pino Ramos-Sosa, ${ }^{2}$ Ismael Rodriguez-Lara ${ }^{3}$ \\ ${ }^{1}$ Departamento de Teoría e Historia Económica, Universidad de Malaga \\ ${ }^{2}$ Departamento de Economía, Universidad Loyola Andalucía \\ ${ }^{3}$ Departamento de Teoría e Historia Económica, Universidad de Granada
}

March 5, 2019

\begin{abstract}
We induce conformity in a binary-decision voting game in which one of the options require certain support (majority, supermajority or unanimity) to be the adopted decision. We consider heterogenous types of voters in that each of them prefer a different outcome in the voting game. We demonstrate theoretically that truthful voting is the unique equilibrium without conformity for each possible voting rule. Introducing conformity enlarges the set of equilibria, which includes voting profiles in which agents do not necessarily vote for their preferred option. If we account for the presence of non-conformist honest voters that vote truthfully for their preferred option, truthful voting is more pervasive for conformist voters in equilibrium. In our setting, the effects of conformity and honest voters on the likelihood of voting truthfully depend on the voting rule that determines whether or not voters are in a decisive group to implement one of the decisions. We provide empirical support for our theoretical predictions by means of a laboratory experiment. Our findings indeed suggest an interplay between the voting rule and the willingness to conform.
\end{abstract}

Keywords: truthful voting, conformity, honest voters, voting rules, experimental evidence.

JEL Class.: C91, C92, D71, D72 
Those are my principles, and if you don't like them... well, I have others.

Groucho Marx.

\section{Introduction}

People frequently face binary decisions that require their opinion: board members choosing whether to accept or reject a proposal (e.g., projects and budgets), senators and congressmen voting on whether to pass a bill, or citizens voting in a referendum. In these cases, agents might have a clear and strong opinion on what the best decision is. If they do not pay attention to anything else, one can expect them to vote instrumentally for the option that best suits their opinions, that is, to vote truthfully. ${ }^{1}$ However, agents may decide to misrepresent their opinion and conform to other agents by voting for the alternative option.

There is mounting evidence that agents possess a desire to conform to others when casting their votes. The literature on bandwagon behavior, for example, highlights that agents may have a desire to vote with the majority group in an election so as to vote for the winning option (Callender, 2007; Morton et al., 2015; Morton and Ou, 2015; Agranov et al., 2017). Arguably, decisions are not always adopted using majority rules, therefore voting for the winning option is not always equivalent to voting with the majority group. The current paper is an attempt to re-examine the idea of conformity to account for decisions that require more than majority to be adopted. The fact that many institutions employ these voting rules indeed calls for modeling the issue of conformity under different voting rules. In some States of the United States, for example, juries require unanimity for finding a defendant guilty. In the Council of the European Union, unanimity is also used for EU membership, while a supermajority is required when the Council votes on a proposal by the Commission or the High Representative of the Union for Foreign Affairs and Security Policy. Our goal is to study whether (and how) different voting rules influence the willingness to conform of agents when they have different views with regard to what constitutes the best decision in the voting game.

To clarify ideas, consider a situation in which five agents have to vote between options $\mathrm{A}$ and B. Agents have instrumental preferences over the two options: two agents prefer option A (type-A agents) and three agents prefer option B (type-B agents). It is common information that option B

\footnotetext{
${ }^{1}$ In a binary-decision voting game, one agent instrumentally prefers A to B if she gets the highest possible payoff when option A is adopted.
} 
requires a certain degree of support (majority, supermajority or unanimity) to be adopted. In this setting, type-B agents are the (only) decisive group under majority because they can implement any of the two options if they coordinate their votes; in fact, type-B agents can implement their preferred choice when voting in bloc. ${ }^{2}$ Under supermajority or unanimity, both type-A and type-B agents are decisive to implement option A, but none of the groups is decisive to implement option $\mathrm{B}$; in fact, any agent is decisive for option A under unanimity, therefore type-A agents can easily guarantee their preferred option in this setting. As a result, one can easily predict that type-A agents will be more likely to conform under simple majority than under unanimity. In this paper, we incorporate these features in a model of normative conformity to derive behavioral predictions that we test using a laboratory experiment. ${ }^{3}$ Our working hypothesis is that agents do not want to vote alone for their preferred option if this is not going to be adopted because they fear social isolation. ${ }^{4}$ Hence, agents will be willing to conform only when they are not pivotal to implement their preferred choice. Further, agents will be willing to express their instrumental preference and vote truthfully if their preferred choice receives the support of at least one other agent (even if they do not vote for the winner in the election).

While there is evidence that voters may have a desire to conform to others, it is also well documented that some agents have a preference for truth-telling, even when doing so is against their own material utility (Sanchez-Pages and Vorsatz, 2007; Lundquist et al., 2009; Abeler et al., 2016). In line with Dutta and Sen (2012) we refer to these agents as honest voters. We assume that honest voters will vote truthfully for their preferred option regardless of the voting rule (Moreno and Ramos-Sosa, 2017). ${ }^{5}$ Our goal is to investigate how the presence of these honest voters affects the

\footnotetext{
${ }^{2}$ Agents can have incentives to form coalitions and coordinate their votes; see, e.g., Morelli (2004), Eguia (2011) and the references therein.

${ }^{3}$ We focus on the idea of normative conformity as the willingness to conform in our model stems from a desire to 'fit in' or be liked. The literature on conformity considers also the possibility that agents follow others because they want to be correct in their decision (informational conformity). Our paper departs from this view because our agents have instrumental preferences over the two options in a way that each type of agent prefers one of the alternatives; i.e., there is no correct decision that will make it optimal for everyone to vote one of the options. Readers interested in informational conformity in voting can consult, among others, Anderson and Holt (1997), Morton and Williams (1999, 2000), Callander (2007), Morton et al. (2012, 2015), Hung and Plott (2001), or Goeree and Yariv (2015).

${ }^{4}$ This resembles the idea in Wright (1990) that "it may be ok to back the loser in a close battle because one has plenty of support in one's position [but voting] for the loser in a landslide could leave the respondent feeling vulnerable and isolated".

${ }^{5}$ In a context with private information, Feddersen and Pesendorfer (1998) or Battaglini et al. (2010) refer to these agents as partisan voters. In their setting, partisan voters will ignore any signal about the relevant state of the world
} 
likelihood that other agents vote truthfully under different voting rules when we induce conformity.

Altogether we study, both theoretically and empirically, how conformity and the presence of honest voters affect agents' tendency towards voting truthfully under different voting rules. We consider three different scenarios. In the first scenario (Baseline), agents have instrumental preferences and do not have any incentives to conform. We show that voting truthfully is the unique undominated Nash equilibrium in this case. In the second scenario (Conformity), all agents exhibit the same degree of conformity. In this case, agents voting truthfully is an undominated Nash equilibrium but there are also several equilibria in which agents do not vote truthfully. In the third scenario (Honest), we consider both type of agents simultaneously; in particular, it is common information that a subset of two type-B agents will vote truthfully for their preferred option, while the other agents have preferences to conform. In this setting, we show that truthful voting remains being an undominated Nash equilibrium and the set of equilibria in which agents do not vote truthfully shrinks, compared with the case where all agents exhibit the same degree of conformity.

To empirically investigate these predictions, we conduct a laboratory voting game using the Induced Value Theory (Smith, 1976). ${ }^{6}$ Our focus is on investigating how inducing a particular way of conformity can affect truthful voting, thus we deliberately decided to employ monetary incentives to induce subjects to have preferences over alternatives that correspond to our theory. ${ }^{7}$ Consistent with our predictions, we find that agents are less likely to vote truthfully when conformity is induced in the experiment. We also find that there is an effect of the voting rule on the likelihood of voting truthfully for each type of agent. In particular, most type-B agents vote truthfully under the majority rule when we induce conformity, while type-A agents are more likely to conform in this case, compared with the baseline. If unanimity is required, there is no effect of inducing conformity on the voting behavior of type-A agents, but type-B agents vote less truthfully in this voting rule, compared with the baseline. In the presence of conformity, type-B agents are also less likely to vote truthfully under unanimity, compared with the majority rule. These experimental findings lend support for the idea that given a voting a rule, agents in a decisive group are less willing to to vote for their preferred option in an election. As voters do not receive any signal in our model, we avoid using their terminology.

${ }^{6}$ Importantly, we do not intend to test the existence or the type of conformity that agents may posses motivations to conform; e.g., ethical preferences or a desire to vote for the winner.

${ }^{7}$ Other papers that induce preferences over outcomes in a voting game include, among others, Gerber et al. (1998), Morton and Williams (1999), Kube and Puppe (2009), Battaglini et al. (2010), Bassi et al. (2011) or Van der Straeten et al. $(2010,2013,2016)$. 
conform. We also find evidence for the hypothesis that honest voters affect the behavior of agents; in particular, we show that truthful voting becomes more pervasive for type-B agents when it is common information that two honest type-B agents will vote truthfully. Finally, we show that the voting rule can affect the average total payoffs and the likelihood of receiving the maximum possible total payoff. For example, we find that conformity has a positive effect on the likelihood of attaining what we can call the truthful outcome, which is the one that would be elected if agents voted truthfully.

The rest of the paper is organized as follows. In Section 2, we discuss the relevant literature on conformity in voting. In Section 3, we present our theoretical model and derive the main predictions. In Section 4, we present the experimental design. We summarize the testable hypotheses in Section 5. The results are discussed in Section 6. Section 7 concludes. All proofs and additional analysis of our data are relegated to the Appendix.

\section{Related Literature}

We investigate the effect of conformity on truthful voting under different voting rules. A number of researchers have studied the extent to which agents vote truthfully (or strategically) under different electoral systems. The main finding is that the degree of uncertainty and the complexity of the voting rule (e.g., the level of strategic computations) play a crucial role on the behavior of voters (see Gerber et al., 1998; Kube and Puppe, 2009; Van der Straeten et al., 2010, 2013, 2016; Esponda and Vespa, 2014; Bassi 2015; Palfrey 2016; Bouton et al. 2017a, 2017b).

In our game, we induce heterogeneous preferences over outcomes in a way that agents have a clear (but distinct) opinion about what is the best option in the election. This, in turn, implies that the willingness to conform does not occur in a setting with asymmetric information in which agents have an incentive to choose the best candidate (e.g., Anderson and Holt, 1997; Hung and Plott, 2001). The closest paper to ours in this literature is Bassi et al. (2011). In their setting, agents are told their types to create an identity for them. As in our model, some agents are part of the majority group and others of the minority group. The main difference lies in the fact that Bassi et al. (2011) provide incentives for agents to deviate from their assigned types to be on the winning side of an election (bandwagoning voting), while we induce conformity in a way that agents would not want to vote alone, unless they are in the winning side. In a way, we relax the assumption about conformity and extend the findings in Bassi et al. (2011) by looking at the effects of different 
voting rules when we induce conformity among agents. Our findings are consistent with the idea that voters may want to vote for the winning option when the adopted decision does not necessarily coincide with that supported by the majority group.

Our results that the willingness to conform depends on the voting rule and the group decisiveness dovetail with the literature exploring the effects of pivot probabilities on voting decisions. ${ }^{8}$ In the model of Tyran (2004) and Feddersen et al. (2009) voters face a tradeoff between voting for their preferred choice (instrumental preference) and one other alternative that is morally superior (expressive preference); and the pivotality of agents is key in explaining their decisions. ${ }^{9}$ Hochtl et al. (2012) is another paper related to ours. They consider two types of agents (rich and poor) who vote for the level of redistribution in a majority setting. The authors find that the composition of the group (whether rich or poor are in the majority group) is decisive in obtaining redistributive results, thereby suggesting the effects of pivotality in a majority setting (see also Bartling et al. 2015). In our setting, the voting rule determines the decisiveness of each group, which seems to play a crucial role on the willingness to conform of agents.

Finally, our model is highly relatd to other papers that study how the presence of honest agents influence voting behavior. Dutta and Sen (2012) show that the presence of these agents can facilitate the implementation of social choice correspondences that satisfy no veto power. Morton et al. (2012), Barberà and Nicolò (2016) or Bouton et al. (2017a) have studied the effect of truthful voting on information aggregation and information disclosure. The fact that voters are aware of the presence of honest voters relates our paper to the study of voting behavior when information about the behavior of other voters is disclosed. In the context of strategic voting, Kube and Puppe (2009) and Esponda and Vespa (2014) argue that being informed about the actual behavior of other voters can facilitate strategic behavior. Our data suggest that being aware of the presence of honest voters affect the willingness to vote truthfully of conformist agents.

\footnotetext{
${ }^{8}$ The participation decision in an election is not only affected by the pivot probability (Feddersen and Sandroni (2006), Levine and Palfrey (2007), Duffy and Travits, (2009)) but can also be influenced by the willingness to conform (Blais and Hortala-Vallve, 2016, 2017; Agranov et al., 2017).

${ }^{9}$ One might think that the preferred choice of agents (i.e., their instrumental preference) is to maximize their material payoff, while the alternative (i.e., their expressive preference) is to maximize the total payoffs or minimize inequality. Tyran and Sausgruber (2006) show that accounting for inequality aversion can explain voting in redistribution problems.
} 


\section{Model}

\subsection{Agents, actions, and preferences}

Consider five agents $N=\{1,2,3,4,5\}$ who have to vote between two options, A and B. Agents 1,2 and 3, that we call type- $B$ agents, receive a positive payment if option B is elected, 0 otherwise. Agents 4 and 5 are called type- $A$ agents and obtain a positive payment if option $\mathrm{A}$ is elected, 0 otherwise. The list of types $t=\left(t_{1}, t_{2}, t_{3}, t_{4}, t_{5}\right)=(B, B, B, A, A)$ is common information, where $t_{i} \in\{A, B\}$ stands for the type of the agent $i \in N$.

Agents vote simultaneously for one of the two options (abstention is not allowed). Let $M_{i}=$ $\{A, B\}$ be the set of messages for agent $i \in N$, where $m_{i}=A\left(m_{i}=B\right)$ stands for agent $i$ voting for option A (option B). Let $M=\times_{i \in N} M_{i}$ be the set of messages and $m_{-i} \in M_{-i}=\times_{j \in N \backslash\{i\}} M_{j}$ be the messages of all agents except $i$. We denote the profile of messages by $m \in M$, while $x \in\{A, B\}$ stands for the adopted decision.

Option $\mathrm{B}$ is adopted if a given number of agents $q \in\{3,4,5\}$ vote for that option, option $\mathrm{A}$ is adopted otherwise. We refer to $q$ as the voting rule, or the threshold needed for option $\mathrm{B}$ to be adopted.

Definition: A $q$-threshold rule $q(m): m \rightarrow x$ is such that, for any $m \in \times_{i \in N} M_{i}$

$$
q(m)= \begin{cases}A & \text { if } \#\left(m_{i}=B\right)<q \\ B & \text { if } \#\left(m_{i}=B\right) \geq q\end{cases}
$$

A 3- (4-) [5-] threshold rule implies that option B requires simple majority (supermajority) [unanimity] to be adopted.

Agents' utility functions consist of two elements: (i) $v\left(t_{i}, q(m)\right.$ ), that depends on the adopted decision and take a positive value $v>0$ if the decision coincides with the type of the agent, 0 otherwise; and (ii) a non-negative function $c(m)$, that depends on the profile of messages announced by the agents. Agents' preferences can then be written as follows:

$$
u\left(t_{i}, q, m\right)=v\left(t_{i}, q(m)\right)+c_{i}(m)
$$

where 


$$
v\left(t_{i}, q(m)\right)=\left\{\begin{array}{ll}
v & \text { if } q(m)=t_{i} \\
0 & \text { if } q(m) \neq t_{i}
\end{array} .\right.
$$

The term $c_{i}(m)$ determines whether or not agents care about other agents' messages. In our baseline scenario, agents obtain an extra payment $c_{i}(m)=c$ for just casting their ballot, thus $c_{i}(m)$ is independent of the profile of messages. In our conformity scenario, we say that agent $i$ conforms to agent $j \in N \backslash\{i\}$ if $m_{i}=m_{j}$. We assume that $c_{i}(m)=c$ when $m_{i}=m_{j}$ for some $j \in N \backslash\{i\}$; otherwise, $c_{i}(m)=0$; i.e., agents obtain the extra payment only if they conform to at least one other agent. ${ }^{10}$ We assume that $v>c(m) \geq 0$ for any $m \in M$ and $i \in N$ to capture the idea that agents attach more weight to obtaining their preferred option; in fact, conformist agents' preferences are lexicographical in the sense that agents always prefer their option to be elected and would conform if they are not pivotal.

To investigate the effects of conformity on voting behavior, we compare the behavior in the baseline and conformity scenario, which differ in the form of the additional payment that agents get for casting their votes. In the baseline scenario, subjects receive the extra payment for casting their votes; i.e., $c(m)=c$ for any $m \in M$, while they receive the additional amount of money only if they vote for the same option as any other member of their group in the conformity scenario. Then, we allow for heterogeneity of types to study how the likelihood of voting truthfully is affected in the presence of honest voters. In our game, we consider two type-B agents (say agents 1 and 2) that vote truthfully for their type. The reason to have two type-B agents as honest voters is twofold. On the one hand, we want to study the effects of honest voters in the presence of conformity. As the payment to conform $c_{i}(m)=c>0$ requires that at least two agents vote the same option, we need at least two honest agents in our model (as we will see below, this is also convenient for experimental purposes). On the other hand, we want to avoid that one of the options is adopted automatically when we account for the presence of honest agents in the voting game. This rules

\footnotetext{
${ }^{10}$ There may be several ways of defining conformity (see Moreno and Ramos-Sosa, 2017). For models that assume that agents experience a benefit or cost depending on whether they vote with the majority or for the winner of the election see, among others, Luzzati (1999); Hung and Plott (2001), Callender (2007), Morton et al. (2012); Battaglini et al. (2010), Bassi et al. (2011), Michaeli and Spiro (2015). In Appendix B, we develop a simple model in which agents may want to conform for efficiency motives (Feddersen et al., 2009) and discuss the extent to which this model reconciles with the observed behaviour. Overall, we find that the efficiency motive is not the main determinant to conform, but voters may have a tendency to vote for the winning option.
} 
out the possibility of having two type-A agents in the role of honest voters, what would result in adopting option $\mathrm{A}$ under the 4-threshold and the 5 -threshold rules. ${ }^{11}$

\subsection{Theoretical predictions}

First, we define the equilibrium concept that we use throughout our paper.

Definition. For any $q \in\{3,4,5\}, m \in M$ is a weakly undominated Nash equilibrium (WUNE) if for any $i \in N$,

(1) $m_{i}$ is not weakly dominated and

(2) for any $m_{i}^{\prime} \in M_{i}, u_{i}\left(t_{i}, q, m_{i}, m_{-i}\right) \geq u_{i}\left(t_{i}, q, m_{i}^{\prime}, m_{-i}\right)$.

Recall that in our baseline scenario, all agents receive $c_{i}(m)=c$ regardless of the profile of messages $m \in M$. Proposition 1 shows that all agents voting truthfully is the unique WUNE for any $q$-threshold rule such that $q \in\{3,4,5\}$. The intuition is that when agents are pivotal, they are better off voting truthfully for their type than voting for the alternative option. In addition, agents are not worse off voting for their type when they are not pivotal, thus voting truthfully is the unique weakly dominant strategy for all agents.

Proposition 1. In the baseline scenario, all agents voting truthfully is the unique WUNE, that is, type- $B$ agents vote for $B$ and type- $A$ agents vote for $A$ for any $q$-threshold rule, where $q \in\{3,4,5\}$ Proof. See Appendix B.

In the conformity scenario, agents obtain the extra payment only if they do not vote alone. Proposition 2 shows that in the conformity scenario, voting truthfully remains as an equilibrium strategy for all the agents. Moreover, there are also other equilibria in which agents vote for an option that does not coincide with their type. Proposition 2 follows from the fact that no message is weakly dominant for any agent for $q \in\{3,4\}$, that type-A agents voting truthfully is a weakly dominant message for $q=5$ and that agents vote truthfully if they are pivotal (if they are not, then conformity prevents agents from voting alone).

Proposition 2. In the conformity scenario, the set of WUNE is given by:

i) all agents vote truthfully for any $q$-threshold rule, where $q \in\{3,4,5\}$

\footnotetext{
${ }^{11}$ Moreno and Ramos-Sosa (2017) provide the number of honest agents that should vote truthfully within a group to guarantee the truthful outcome. Their results hold for any voting rule $q \in\{1, n\}$, any number of agents $n$ and any list of agents' types $t$.
} 
ii) all agents vote for $A$ for any $q$-threshold rule, where $q \in\{3,4,5\}$

iii) all agents vote for $B$ for any $q$-threshold rule such that $q \in\{3,4\}$, and

iv) all type- $A$ agents and one of the type- $B$ agents vote for $A$ (the other two type- $B$ agents vote for

B) for the 4-threshold rule.

Proof. See Appendix B.

Finally, we consider two type-B agents (say agents 1 and 2) that are honest voters and vote truthfully for their preferred option. We characterize the set of WUNE in our next proposition. The result follows from the fact that voting truthfully is a weakly dominant message for the type-B agent for any $q \in\{3,4,5\}$ and for the type-A agent when $q=5$. Also, by conformity, type-A agents vote for the same option in any WUNE when $q \in\{3,4\}$.

Proposition 3. In the honest scenario, the set of WUNE is given by:

i) all agents voting truthfully for any $q$-threshold rule such that $q \in\{3,4,5\}$, and

ii) all agents voting for $B$ for any $q$-threshold rule such that $q \in\{3,4\}$.

Proof. See Appendix B.

These theoretical results show that conformity enlarges the set of equilibria, while the presence of honest voters reduces it. In the next section, we outline our experimental design that is designed to test our theory.

\section{Experimental design}

A total of 390 subjects were recruited to participate in our computerized sessions (Fischbacher, 2007). Subjects were Economics or Business students from the undergraduate population of the Universidad de Valencia, with no previous experience in similar experiments. Subjects were invited to participate in our experiment using the recruitment system of the laboratory (LINEEX).

We ran a total of 13 sessions with 30 subjects each. At the beginning of each session, subjects were randomly assigned a type (Player A or Player B), which was held constant throughout the session. Subjects were told that they were in a group of 5 subjects. It was common information that each group consisted of 2 Players A and 3 Players B.

Our experiment relies on a between-subjects design (i.e., subjects only participate in one of the three possible treatments). Each treatment is designed to mimic a theoretical scenario as follows: 
- Baseline (BL, 120 subjects, 24 groups). Subjects received 75 ECUs if the adopted decision coincided with their own type. Subjects received an additional amount of 25 ECUs, regardless of the option for which they decided to vote.

- Conformity (CON, 120 subjects, 24 groups). Subjects received 75 ECUs if the adopted decision coincided with their own type. They received the additional payoff of 25 ECUs only if their vote coincided with that of any other subject in their group.

- Honest (HON, 150 subjects, 30 groups). Payoffs were as in the conformity treatment, but it was common information that 2 subjects in the role of Player B would be given no option but to vote for option B. These forced subjects play the role of honest voters. ${ }^{12}$

To investigate the effect of the $q$-threshold rules on behavior, we employ the strategy method (Selten, 1967; Brandts and Charness, 2011). In each treatment, subjects were asked to vote between option A and option B in three different scenarios, depending on whether option $\mathrm{B}$ required $q=$ $\{3,4,5\}$ votes to be the adopted decision. In our experiment, we control for the order of $q$ and balance the number of observations across sequences of decisions; e.g., we had the same number of observations for sequences $3,4,5$ and $5,3,4$.

Instructions were read aloud by the session monitor, and subjects were allowed to ask any question in private before starting the experiment. We minimized the probability of subjects failing to understand how payoffs were generated using a pre-experimental quiz, in which subjects were asked to compute the payoffs of randomly generated examples.

At the end of the experiment, one decision was randomly selected for payment (Azrieli et al. 2018). Each session lasted for approximately 1 hour, and the subjects received approximately 7.5 Euros for participating (10 ECUs $=1$ Euro). The experiment included an additional phase in which individual characteristics (e.g., age, gender, cognitive abilities,...) were elicited. We will use these variables as controls in our econometric analysis. Our questionnaire, together with a translated version of our original instructions, is presented in Appendix A.

\footnotetext{
${ }^{12}$ We decided to have two subjects instead of the computer in the role of honest voters to avoid any concern about social preferences; e.g., type-A agents can vote differently depending on whether they impose an externality on the computer or on another human subject. For the effects of social preferences on bandwagon voting, see Morton and Ou (2015) or Corazzini and Greiner (2007).
} 


\section{Testable Hypotheses}

We summarize our theoretical predictions in Table 1, which provides the set of WUNE for each of the three scenarios. In the first treatment $(\mathrm{BL})$, subjects are expected to vote truthfully regardless of the voting rule (Proposition 1).

Prediction 1. Subjects vote truthfully in the baseline treatment, and the voting rule does not have any effect on their voting behavior.

Given our theoretical results (Proposition 2), we predict that truthful voting will be less pervasive when we induce conformity in the CON treatment; in fact, Table 1 shows that the set of WUNE enlarges in this treatment.

Prediction 2. The presence of conformity will reduce the likelihood of voting truthfully, compared with the baseline treatment.

\begin{tabular}{|c|c|c|c|c|c|c|}
\hline & Baseline (BL) & & Conformity (CON & & Honest (HON) & \\
\hline$q=3$ & $(\mathrm{~B}, \mathrm{~B}, \mathrm{~B} ; \mathrm{A}, \mathrm{A})$ & {$[B]$} & $\begin{array}{l}(\mathrm{B}, \mathrm{B}, \mathrm{B} ; \mathrm{A}, \mathrm{A}) \\
(\mathrm{B}, \mathrm{B}, \mathrm{B} ; \mathrm{B}, \mathrm{B}) \\
(\mathrm{A}, \mathrm{A}, \mathrm{A} ; \mathrm{A}, \mathrm{A})\end{array}$ & $\begin{array}{l}{[B]} \\
{[B]} \\
{[A]}\end{array}$ & $\begin{array}{l}(\mathrm{B}, \mathrm{B}, \mathrm{B} ; \mathrm{A}, \mathrm{A}) \\
(\mathrm{B}, \mathrm{B}, \mathrm{B} ; \mathrm{B}, \mathrm{B})\end{array}$ & $\begin{array}{l}{[B]} \\
{[B]}\end{array}$ \\
\hline$q=4$ & $(\mathrm{~B}, \mathrm{~B}, \mathrm{~B} ; \mathrm{A}, \mathrm{A})$ & {$[A]$} & $\begin{array}{l}(\mathrm{B}, \mathrm{B}, \mathrm{B} ; \mathrm{A}, \mathrm{A}) \\
(\mathrm{B}, \mathrm{B}, \mathrm{B} ; \mathrm{B}, \mathrm{B}) \\
(\mathrm{A}, \mathrm{A}, \mathrm{A} ; \mathrm{A}, \mathrm{A}) \\
(\mathrm{A}, \mathrm{B}, \mathrm{B} ; \mathrm{A}, \mathrm{A})^{*}\end{array}$ & $\begin{array}{l}{[A]} \\
{[B]} \\
{[A]} \\
{[A]}\end{array}$ & $\begin{array}{l}(\mathrm{B}, \mathrm{B}, \mathrm{B} ; \mathrm{A}, \mathrm{A}) \\
(\mathrm{B}, \mathrm{B}, \mathrm{B} ; \mathrm{B}, \mathrm{B})\end{array}$ & $\begin{array}{l}{[A]} \\
{[B]}\end{array}$ \\
\hline$q=5$ & $(\mathrm{~B}, \mathrm{~B}, \mathrm{~B} ; \mathrm{A}, \mathrm{A})$ & {$[A]$} & $\begin{array}{l}(\mathrm{B}, \mathrm{B}, \mathrm{B} ; \mathrm{A}, \mathrm{A}) \\
(\mathrm{A}, \mathrm{A}, \mathrm{A} ; \mathrm{A}, \mathrm{A})\end{array}$ & $\begin{array}{l}{[A]} \\
{[A]}\end{array}$ & $(\mathrm{B}, \mathrm{B}, \mathrm{B} ; \mathrm{A}, \mathrm{A})$ & {$[A]$} \\
\hline
\end{tabular}

Notes. Recall that each group is formed by three type-B agents and two type-A agents; i.e., $t=(\mathrm{B}, \mathrm{B}, \mathrm{B} ; \mathrm{A}, \mathrm{A})$. The first three elements in each profile of messages above indicate the equilibrium votes of type-B agents; while the remaining two elements stand for the vote of the type-A agents. To simplify notation, we do not present all the WUNE for the different types of voters within a group; e.g., (A, B, B; A, A) is equivalent to (B, $\mathrm{A}, \mathrm{B} ; \mathrm{A}, \mathrm{A})$ or (B, B, A; A, A). In all of these cases, all type-A agents vote for A and one type-B agent votes for A (the other type-B agent votes for B). The equilibrium outcome appears in brackets and italics.

Table 1: Set of WUNE in each of the three scenarios for each possible $q$-threshold rule.

To make predictions on the interplay between conformity and truthful voting under different voting rules, we refer to the idea of group decisiveness. We say that a group is decisive to implement one option if the members of the group can coordinate their votes and implement this option when voting as a bloc. When decisions are taken by simple majority, type-B agents have group decisiveness 
because they can guarantee any of the two options if they vote as a bloc. In the 3-threshold rule, the equilibria in the CON treatment is such that either all agents vote truthfully or they all vote unanimously for the same option. Because group B is decisive, we expect for option B to be elected; either because agents all vote unanimously for option B or because they vote truthfully. Arguably, strategic uncertainty and coordination problems are likely to play a role in this setting. ${ }^{13}$ If type-B agents vote as a bloc, then type-A agents face a coordination problem. If both type-A agents vote truthfully, they will obtain the additional payoff for conformity, but this can be perceived as being more risky than simply voting for option B. In fact, if one of the type-A agent believes that the other one is going to conform, she should conform as well; note that a type-A agent voting truthfully is never an equilibrium. As a result, we expect that CON will reduce the likelihood of truthful voting in the 3-threshold rule, especially for type-A agents. In the 4-threshold rule, both groups are decisive to implement option A. Type-A agents can then be affected when we introduce conformity but they can guarantee their preferred choice if they vote as a bloc; in fact, most of the equilibrium configuration result in option A being elected. We thus expect that type-A agents will be less likely to conform in this case, compared with the 3 -threshold rule. Finally, any agent can implement option $\mathrm{A}$ in the 5 -threshold rule thus we expect that type-A agents will vote truthfully in this setting; i.e., we expect that inducing conformity will have no effect on type-A agents, compared with the BL treatment. Type-B agents may anticipate that option A will be elected under the 5-threshold rule, thus type-B agents may be more willing to conform under this rule so as to avoid the coordination problem above. An additional concern to conform is the willingness to vote for the winner of the election. Although we do not provide incentives for agents to vote for the winning option as it occurs in Hung and Plott (2001) or Bassi et al. (2011), the bandwagon effect may also influence voting behavior; in fact, this effect emerges endogenously in our setting. We argue that agents will be less likely to vote truthfully when they are not in the decisive group because they anticipate that agents in the decisive group will implement their preferred outcome.

Prediction 3. When we introduce conformity, the behavior of type-A (type-B) agents will be mainly affected in the 3-threshold (5-threshold) rule, where they will reduce the likelihood of voting truthfully.

Our prediction that type-A agents might vote more truthfully in the 5-threshold rule, compared with the 3- and 4-threshold rules, highlights the importance of the group decisiveness on the

\footnotetext{
${ }^{13}$ For the role of strategic uncertainty see also Bouton et al. (2017a).
} 
willingness to vote truthfully under conformity. Along these lines, type-B agents can anticipate that increasing the voting rule makes it more difficult for option B to be chosen in any possible setting. Type-B agents can then be less likely to vote truthfully when the voting rule becomes more stringent in CON. Another way of looking at the effect of conformity under different voting rules is then as follows:

Prediction 4. There is an effect of the q-threshold rule on the agents' likelihood of voting truthfully when we induce conformity. Type-A (type-B) agents will be more (less) likely to vote truthfully when the value of $q$ increases.

Next, we look at the behavior of agents when we introduce honest agents. Compared with the CON treatment, the equilibrium strategies reduce to only two in the HON treatment: all agents voting truthfully and all agents unanimously voting for B, except for the 5 -threshold rule, where all agents vote truthfully. This, in turn, implies that the type-B agent that is not forced to vote truthfully has a dominant strategy in equilibrium, which is voting truthfully.

Prediction 5. In a conformity setting, the presence of two honest type- $B$ agents will increase the likelihood that the other type-B agent will vote truthfully.

When we compare CON and HON, our theoretical predictions in Table 1 seem to suggest that the behavior of the type-B agent will be mainly affected in the 4 -and the 5 - threshold rules. In the 3-threshold rule, type-B agents are expected to vote truthfully in the CON because they are in the decisive group, thus we expect that the presence of honest agents will not have a major effect in the willingness to vote truthfully, compared with the CON. In the 4-threshold rule, the set of equilibria reduces in the HON to all agents voting truthfully or voting for option B; thus we expect that type-B agent will be more willing to vote truthfully in the presence of honest agents under supermajority; in fact, there is no equilibria in which type-B agents vote for option $\mathrm{A}$. A similar argument applies in the 5 -threshold rule, when the equilibrium reduces to all agents voting truthfully.

Finally, we posit a prediction on the adopted decision. If agents voted truthfully, option B would be elected under unanimity, while option A would be elected under majority and unanimity. We consider this truthful outcome as the natural benchmark to study the effect of conformity and honest voters. Our predictions above suggest that conformity will facilitate the election of the truthful outcome because type-A (type-B) agents will be more likely to vote for option B (option 
A) as the value of $q$ increases when we induce conformity. The introduction of honest voters is expected to dilute the willingness to conform by fostering the willingness to vote truthfully. This, in turn, may have a negative effect on the likelihood of attaining the truthful outcome.

Prediction 6. Conformity has a positive effect on the likelihood of attaining the truthful outcome. The introduction of honest voters facilitates truthful voting, and this will hinder the likelihood of attaining the truthful outcome.

\section{Results}

This section presents our experimental evidence. Section 6.1 focuses on the first two treatments (BL vs. CON) to show $i$ ) the effects of conformity on the likelihood of voting truthfully, and $i i$ ) the influence of the voting rule in each treatment. In Section 6.2, we assess whether the presence of honest voters influences agents' voting behavior in a conformity setting (CON vs. HON). We provide some insights into efficiency and evaluate our findings in terms of social welfare in Section 6.3.

\subsection{On the effects of conformity}

Figure 1 depicts the effect of conformity by plotting the average likelihood of voting truthfully in BL and CON, separately for each type of agent. Error bars reflect standard error of the mean. The results disaggregated by voting rule are presented in Table 2 below the figure. This includes the results of our $\chi^{2}$ non-parametric analysis. ${ }^{14}$

In line with our Prediction 1, we find that the $q$-threshold rule does not affect the willingness to vote truthfully in the BL treatment, but it affects the behavior of agents in the CON treatment (Prediction 4) (see Table 3 below for further discussion). As for our Prediction 2, we observe that the presence of conformity decreases the average frequency of voting truthfully both for type-A agents (from 0.75 to $0.60, p=0.003$ ) and type-B agents (from 0.87 to $0.75 p<0.001$ ).

There seems to be a relationship between the group decisiveness and the willingness to conform; in fact, conformity decreases the likelihood of type-A agents voting truthfully for any possible $q$-threshold rule, with the smallest effect when $q=5$. For type-B agents, conformity decreases

\footnotetext{
${ }^{14}$ The reported $p$-values refer to one-sided alternative. Our findings are robust to other statistical analyses; e.g., test of proportions, Mann-Whitney-Wilcoxon test or robust rank-order test (Feltovich, 2003).
} 
Type-A

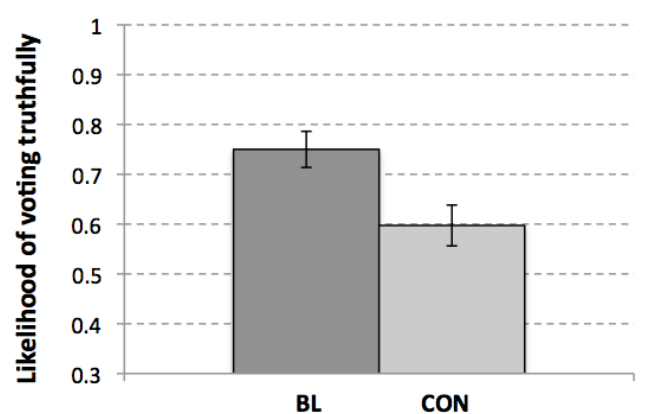

BL

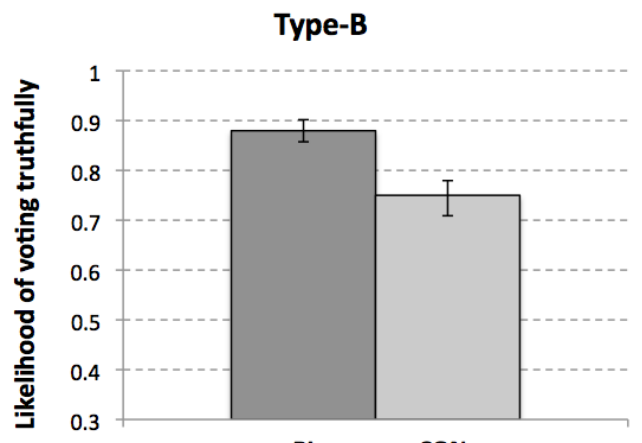

BL

CON

Figure 1: Effect of conformity on the likelihood of voting truthfully.

Table 2: Frequency of truthful voting in BL and CON for each possible $q$-threshold rule.

\begin{tabular}{ccccccc} 
& \multicolumn{3}{c}{ Type-A } & \multicolumn{3}{c}{ Type-B } \\
\hline$q$-threshold rule & BL & CON & $p$-value & BL & CON & $p$-value \\
\hline \hline$q=3$ & 0.71 & 0.46 & $(0.006)$ & 0.90 & 0.94 & $(0.34)$ \\
$q=4$ & 0.85 & 0.71 & $(0.042)$ & 0.90 & 0.74 & $(0.004)$ \\
$q=5$ & 0.69 & 0.62 & $(0.52)$ & 0.83 & 0.57 & $(0.001)$ \\
\hline$N$ & 48 & 48 & & 72 & 72 & \\
\hline
\end{tabular}

the likelihood of voting thrufully for each possible $q$-threshold rule, except when $q=3$. These findings are in line with our Prediction 3 that suggests a relationship between coordination problems, strategic uncertainty and truthful voting. We find that agents are more likely to vote truthfully in those $q$-threshold rules under which they are in the decisive group to implement their preferred choice ( $q=5$ for type-A agents and $q=3$ for type-B agents). In fact, less than half of the type-A agents vote truthfully when $q=3$ in CON and roughly 60 percent of type-B agents vote truthfully when $q=5$ in $\mathrm{CON}^{15}$

We employ an econometric approach to support our predictions. We estimate the effect of conformity on the likelihood of voting truthfully using a logit model (Appendix C contains the details

\footnotetext{
${ }^{15}$ This result is robust when we perform the analysis at the individual level (see Appendix C) and it is much in line with the comment made by Charles Plott to the authors in the sense that "the votes under majority-rule institutions are typically overwhelming majorities because the minority, when anticipating a loss on the vote, just go along with the majority."
} 
of our econometric approach, including robustness checks). Figure 2 depicts the average marginal effect of conformity, together with the $95 \%$ confidence intervals, for each possible $q$-threshold rule. We find that conformity reduces the likelihood of type-A agents voting truthfully by 22 percent when $q=3(p=0.017)$ and by roughly 13 percent when $q=4(p=0.003)$. The effect of conformity is not significant for type-A agents when $q=5$. For type-B agents, conformity has no effect when $q=3$, but it reduces the likelihood of voting truthfully by 11 percent when $q=4(p=0.004)$ and by 32 percent when $q=5(p<0.001)$.
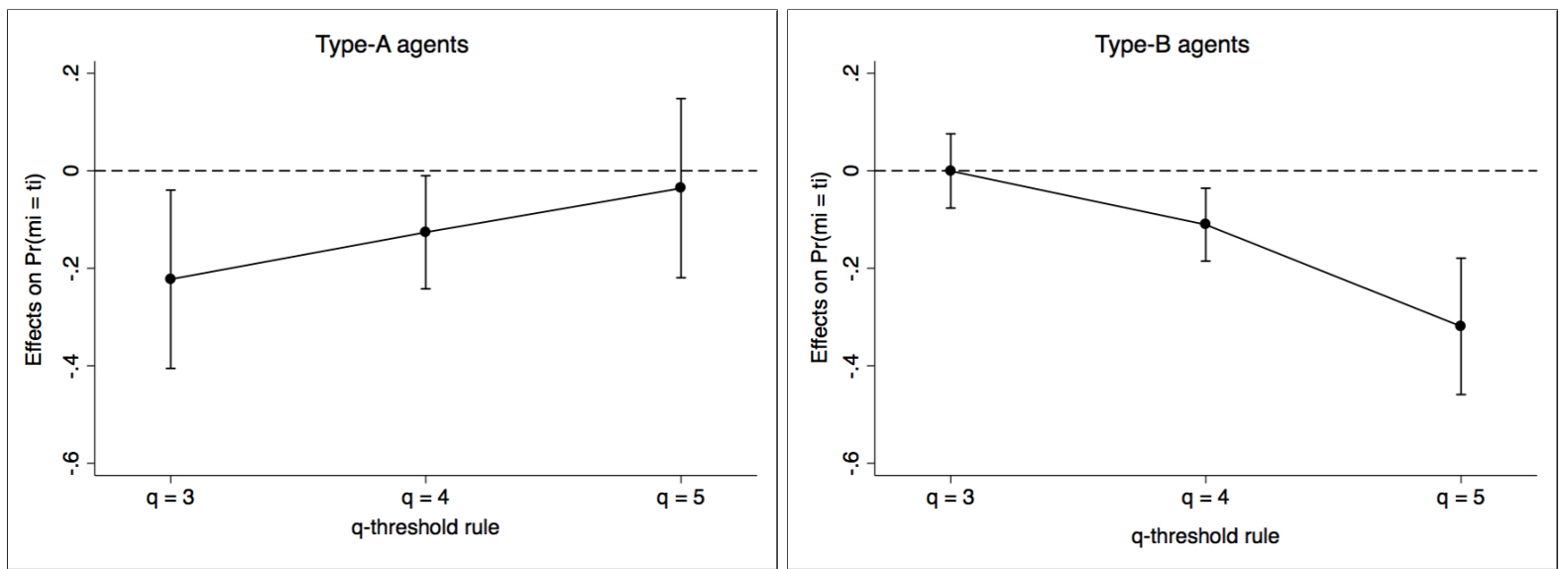

Figure 2: Marginal effects of conformity on the likelihood of voting truthfully after logit specification.

We summarize these findings as follows:

Observation 1. Conformity decreases the likelihood of voting truthfully. The effects of conformity depend on the q-threshold rule.

To study the effect of the $q$-threshold rule on the willingness to vote truthfully in BL and CON, we estimate a logit regression for each treatment and each type of agent, separately. We include dummies $d_{q 4}$ and $d_{q 5}$ for the 4 -threshold and 5-threshold rules, respectively (the omitted category is the 3-threshold rule). The estimated marginal effects are reported in Table 3. The controls include the variables elicited in our questionnaire.

We find no effect of the $q$-threshold rule on the likelihood of voting truthfully in the BL treatment (Prediction 1). For the CON treatment, our findings suggest that type-A agents are more likely to vote truthfully as the voting rule becomes more stringent, whereas type-B agents appear to vote less truthfully as the voting rule becomes more stringent. This confirms the relationship between the likelihood of voting truthfully and the group decisiveness (Prediction 4). 
Table 3: Marginal effects of the $q$-threshold rule on the likelihood of voting truthfully in BL and CON.

\begin{tabular}{lcccc}
\hline & \multicolumn{2}{c}{ Type-A } & \multicolumn{2}{c}{ Type-B } \\
\hline & BL & CON & BL & CON \\
\hline$\left(d_{q 4}=1\right.$ if $\left.q=4\right)$ & 0.149 & $0.25^{* * *}$ & 0.000 & $-0.208^{* * *}$ \\
& $(0.09)$ & $(0.10)$ & $(0.05)$ & $(0.06)$ \\
$\left(d_{q 5}=1\right.$ if $\left.q=5\right)$ & -0.021 & 0.167 & -0.069 & $-0.375^{* * *}$ \\
& $(0.10)$ & $(0.10)$ & $(0.06)$ & $(0.06)$ \\
Controls & Yes & Yes & Yes & Yes \\
Wald $\chi^{2}-$ test & $25.73^{* *}$ & $19.55^{*}$ & 19.43 & $29.19^{* * *}$ \\
Pseudo- $R^{2}$ & 0.09 & 0.08 & 0.08 & 0.17 \\
Observations & 144 & 144 & 216 & 216 \\
\hline
\end{tabular}

Note: Significance at the ${ }^{*} 10 \%,{ }^{*} 5 \%,{ }^{* * *} 1 \%$ level.

Observation 2. i) The q-threshold rule has no effect on the likelihood of voting truthfully when there is no conformity. ii) Under conformity, the q-threshold rule has an effect on the likelihood of voting truthfully, with agents being more likely to vote truthfully when their group is decisive for implementing their preferred option.

One final aspect that is worth mentioning is that our model predicts that all agents will vote truthfully in the BL treatment. We find, however, that type-B agents are more likely to vote truthfully than type-A agents in the BL treatment (0.75 vs 0.83$)$. This is an interesting finding in line with Bassi et al. (2011), who report that members in the minority group do not vote truthfully if decisions are made by majority rule. In Appendix B, we develop a model of conformity that incorporates the possibility that type-A agents are efficiency-oriented and vote for option B because it maximizes the total payoffs. Our model predicts that type-A agents will vote for option B in equilibrium, regardless of the voting rule. This does not seem to reconcile with our data; e.g., $70 \%$ of type-A agents vote truthfully in the 3 -threshold rule. ${ }^{16}$ Our interpretation is that the group

\footnotetext{
${ }^{16}$ We cannot rule out the possibility that some type-A agents fail to vote truthfully in the BL treatment because there is noise in our data. We believe, however, that our finding that more than 70 percent of the type-A agents vote for their preferred option in the BL provide enough support for the hypothesis of truthful voting, especially when we compare these figures with the behaviour of subjects in other voting experiments; e.g., in a simultaneous-voting game, Esponda and Vespa (2014) find that nearly 80 percent of subjects fail to behave optimally, because they have problems to anticipate the behavior of other voters. Van der Straeten et al. (2010) also find evidence of irrational
} 
decisiveness (or the willingness to vote for the winner) is the major force in driving this behavior.

\subsection{On the effects of honest voters}

We replicate our previous analysis to show the effect of honest voters on the likelihood of voting truthfully. The results are summarized in Figure 3 and Table 4, which reports the behavior of agents who were allowed to vote for option A or B in the experiment; i.e., honest voters are excluded from the analysis.

Type-A

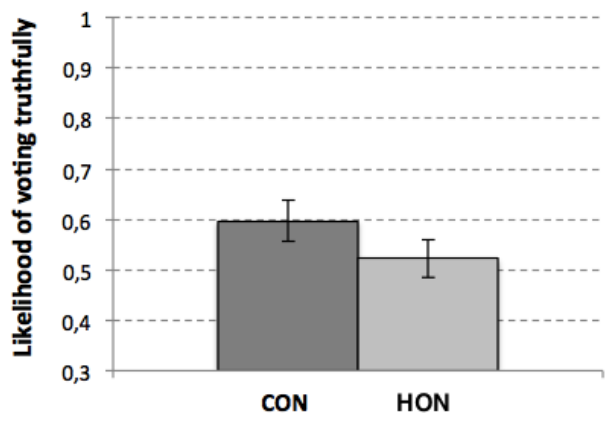

Type-B

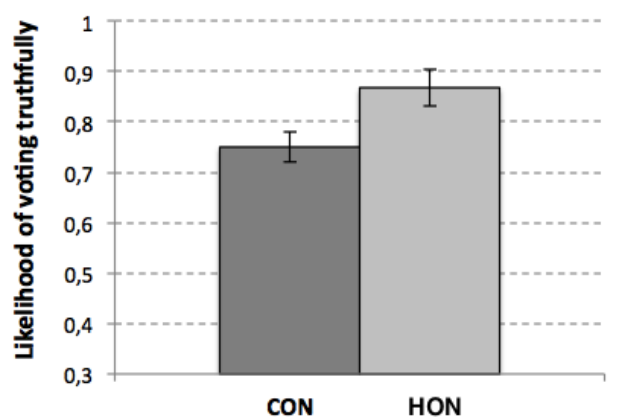

Figure 3: Effect of honest voters on the likelihood of voting truthfully.

Table 4: Frequency of truthful behavior in CON and HON for each possible $q$-threshold rule.

\begin{tabular}{ccccccc} 
& \multicolumn{3}{c}{ Type-A } & \multicolumn{3}{c}{ Type-B } \\
\hline$q$-threshold rule & CON & HON & $p$-value & CON & HON & $p$-value \\
\hline \hline$q=3$ & 0.46 & 0.35 & $(0.25)$ & 0.94 & 0.97 & $(0.64)$ \\
$q=4$ & 0.71 & 0.55 & $(0.046)$ & 0.74 & 0.97 & $(0.004)$ \\
$q=5$ & 0.62 & 0.67 & $(0.65)$ & 0.57 & 0.67 & $(0.36)$ \\
\hline$N$ & 48 & 30 & & 72 & 60 & \\
\hline
\end{tabular}

In line with our Prediction 5, the presence of honest voters increases the likelihood of type-B agents voting truthfully (from 0.75 to $0.86, p=0.012$ ), but the effect seems to be driven by the response of type-B agents when $q=4$. As for type-A agents, the presence of honest voters seems to decrease their likelihood of voting truthfully (from 0.60 to 0.52 ), especially in this case.

behavior or noise in that some subjects fail to vote for the closest candidate to their position in the second round of the election in a two-round majority voting rule. 
We undertake an econometric approach to test of predictions. In Figure 4, we display the marginal effects for the effects of honest voters after controlling for individual heterogeneity (see Appendix C for further details).
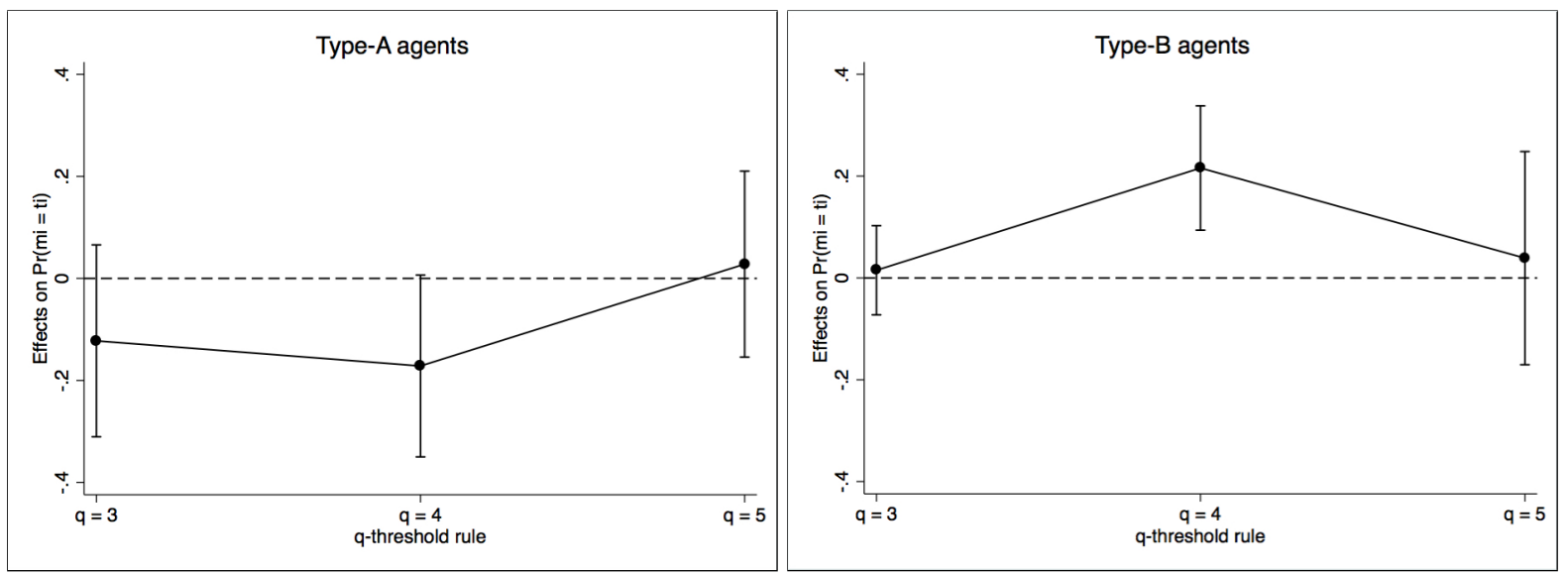

Figure 4: Marginal effects of honest voters on the likelihood of voting truthfully after logit specification.

When we estimate the average effect of the presence of honest voters on the likelihood of voting truthfully, we find that this effect is insignificant for type-A agents $(\mathrm{ME}=-0.089, p=0.12)$ but it is significant for type- $\mathrm{B}$ agents $(\mathrm{ME}=0.090, p=0.043)$. Our estimates in Figure 4 indicate that the presence of honest voters affects differently to agents type-A and type-B agents depending on the threshold rule. In particular, our data suggest that type-A agents reduce their likelihood of voting truthfully by 12 percent when $q=3(p=0.20)$ and by 17 percent when $q=4$. The likelihood increases by roughly 3 percent when $q=5$. As for type-B agents, there is a positive effect of the presence of honest voters on the likelihood of type-B agents voting truthfully, especially when $q=4$. In this setting, the presence of honest voters increases the likelihood of the type-B agent voting truthfully in 22 percent $(p=0.001)$. These findings are consistent with our Prediction 5 , except for the fact that we expected also a positive effect of honest voters on the likelihood of the type-B agent voting truthfully in the 5 -threshold rule. One possible explanation is that type-B agents anticipate that option A will be the winning option and prefer to vote for it, even though this is not associated to any financial incentive.

Observation 3. When agents know that two type-B (honest) agents will vote truthfully, the likelihood of voting truthfully increases for the remaining type-B agent under supermajority.

For the sake of completeness, we examine the effect of the $q$-threshold rule on the likelihood 
of voting truthfully in HON. Using a logit model with dummy variables for the threshold rules, we find that type-A agents are more likely to vote truthfully when $q=4$ and $q=5$, compared with $q=3(\mathrm{ME}=0.20, p=0.029$, and $\mathrm{ME}=0.317, p=0.001$, respectively); hence, the $q$-threshold rule has a positive effect on the likelihood of type-A agents voting truthfully. For type-B agents, there is no effect of the $q$-threshold rule when we compare the likelihood of voting truthfully in $q=3$ and $q=4$, but type-B agents are less likely to vote truthfully in the HON treatment when $q=5(\mathrm{ME}=0.310, p<0.001)$.

\subsection{Social welfare}

Our previous findings suggest that conformity decreases truthful voting, whereas the presence of honest agents fosters it. How do these results translate into efficiency gains or losses? In this section, we look at the issue of social welfare from three different perspectives. First, we compute the average expected payoff to see how much money subjects participating in our experiment get in each treatment for each $q$-threshold rule. Second, total payoffs are maximized when option B is adopted; in fact, subjects will get a maximum of 350 ECUs in this setting. ${ }^{17}$ If agents care about the welfare of the group (Coate and Conlin, 2004; Feddersen et al., 2009; Feddersen and Sandroni, 2006), they will vote to maximize the likelihood of getting the maximum payoff for the group. Finally, one might look at the likelihood of attaining the truthful outcome, which is the one that would be elected if agents voted truthfully; i.e., the truthful outcome is option B in the 3-threshold rule and option A in the 4- and the 5-threshold rules (Moreno and Ramos-Sosa, 2017).

Our welfare analysis in Table 5 look at these three issues. Our approach is to use the behavioral data in each treatment to form all possible configurations of groups consisting of three type-B agents and two type-A agents. Given our data, we can form a total of 961,056 different groups in BL and CON and (at least) 53,100 groups in HON (when we do not combine the behavior of different subjects in the role of forced type-B voters). To obtain the figures in Table 5 we consider the likelihood that type-A and type-B agents vote for each alternative for each possible $q$-threshold rule. For example, we know that type-A agents vote for option A with 0.46 probability in the BL

\footnotetext{
${ }^{17}$ Type-B agents are in the majority group, thus all of them will receive 100 ECUs if option $\mathrm{B}$ is elected. The remaining 50 ECUs are obtained by type-A agents under different conditions. For example, type-A agents get the 50 ECUs in BL, regardless of their votes. If $q=3$, each type-A agent in CON can obtain the 25 ECUs if they both vote truthfully or if they both conform. If $q=5$, then type-A agents need to conform to attain the maximum possible payoff for the group. If one of the agents votes for A, type-A agents will get 75 or 100 ECUs, but type-B agents will be worse off and the total surplus will not be maximized.
} 
Table 5: Efficiency and total surplus

\begin{tabular}{lccc}
\multicolumn{1}{c}{ BL } & CON & HON \\
\hline \hline A. Average total payoff & & \\
$q=3$ & 338.9 & 335.4 & 338.4 \\
$q=4$ & 290.6 & 285.4 & 314.0 \\
$q=5$ & 279.1 & 269.3 & 272.2 \\
\multicolumn{5}{r}{ B02.9 } & 296.7 & 308.2 \\
B. Likelihood of receiving the highest possible payoff (350 ECUs) \\
$q=3$ & 0.85 & 0.50 & 0.54 \\
$q=4$ & 0.21 & 0.03 & 0.20 \\
$q=5$ & 0.06 & 0.03 & 0.07 \\
\multicolumn{4}{c}{ C. Likelihood of attaining the truthful outcome } \\
$q=3$ & 0.85 & 0.96 & 0.27 \\
$q=4$ & 0.79 & 0.76 & 0.32 \\
$q=5$ & 0.95 & 0.97 & 0.93 \\
& 0.86 & 0.90 & 0.75 \\
\hline
\end{tabular}

when $q=3$, while type-B agents vote for option $\mathrm{B}$ with 0.94 probability in this setting. This, in turn, implies that the probability of observing all agents voting for B when we consider all possible groups is $0.54^{2} 0.94^{3}=0.24$. We follow this procedure to obtain the likelihood of each possible configuration of votes in each of the treatments and for each voting rule; e.g., (A, A; A, A, A), (A, $\mathrm{A} ; \mathrm{A}, \mathrm{A}, \mathrm{B}), \ldots,(\mathrm{B}, \mathrm{B} ; \mathrm{B}, \mathrm{B}, \mathrm{B})$.

Panels A and B of Table 5 show that the average expected payoff and the likelihood of receiving the highest possible payoff decreases with the $q$-threshold rule, in every possible treatment. As for the truthful outcome, we show in Panel C that it is more likely to implement option B (A) in the 3 -threshold rule (4-threshold rule), in every possible treatment.

When we compare across treatments, Table 5 shows that CON results in a smaller average expected payoff and a lower likelihood of receiving the highest possible payoff for any possible $q$-threshold rule, compared with the BL treatment. There is a positive effect of conformity on the likelihood of obtaining the truthful outcome (0.86 vs 0.90) (Prediction 6$)$. This occurs especially if $q=3$, when type-A agents tend to vote for option B. Efficiency improves in HON, as the expected 
payoff and the likelihood of maximizing the total payoff increase for each possible $q$-threshold rule. The results are especially remarkable when $q=4$ (0.03 vs 0.20$)$. This occurs because the presence of honest voters has a significant effect on type-A and type-B agents especially in this case (see Table 4). Overall, the effect of HON on implementing the truthful outcome is negative $(0.90 \mathrm{vs}$ 0.75) (Prediction 6). The negative effect of HON is mainly driven by the behaviour of the type-B agent who can vote for any option. When $q=4$ this agent is very likely to vote truthfully for B, but we do not observe this behavior when $q=5$.

Observation 4. i) The likelihood of maximizing total payoffs is higher when there is no conformity, but conformity has a positive effect on the likelihood of attaining the truthful outcome, especially under the majority rule. ii) With the presence of honest voters, the likelihood of maximizing total payoffs increases, while the likelihood of attaining the truthful outcome decreases, especially under supermajority. iii) In every scenario, the likelihood of maximizing the total surplus decreases as the $q$-threshold rule becomes more stringent and, iv) the truthful outcome is more likely under majority and unanimity.

\section{Concluding Remarks}

This paper studies the effects of conformity and information in a binary-decision voting game in which agents are heterogeneous with respect to their preferred outcome. We show that conformity reduces the likelihood of truthful voting for all agents. When agents are informed that two type-B agents will vote truthfully, we expect the other type-B agent to also vote truthfully. Our experimental data support these predictions and highlight the importance of the voting rule in driving the results. In particular, we find that the willingness to vote truthfully and the effects of honest agents depend upon the voting rule and the group decisiveness, thereby suggesting an interplay between the voting rule and the willingness to conform.

Our paper provides a set of important experimental findings regarding the relevance of voting rules for truthful voting. Overall, there is no wide consensus indicating the appropriate voting rule, which depends on the issue at hand and the features of the voting procedure. The theoretical work of Feddersen and Pesendorfer (1998) pleads for the use of the majority rule instead of the unanimity rule in a Condorcet-winner setting (for further discussion on this topic see Guarnaschelli et al., 2000; Anderson et al., 2015; Bouton et al., 2017a). In a model with private information, 
Barberà and Nicolò (2016) and Rivas and Rodriguez-Alvarez (2017) find that informed agents are more prone to disclose truthful information to uninformed agents under the majority rule, while Moreno and Ramos-Sosa (2017) suggest that the decision in equilibrium are more likely to differ from that obtained by truthful voting using the majority rule compared with the unanimity rule. Our results contribute to this literature by providing experimental evidence on the effects of different voting rules on behavior in a setting with conformity. Our result that the presence of honest voters can also affect voting behavior and improve efficiency is in line with the current discussion in Dutta and Sen (2012), Barberà and Nicolò (2016), Rivas and Rodríguez-Alvarez (2017), Battaglini et al. (2010) or Moreno and Ramos-Sosa (2017), who investigate how the presence of agents that always vote truthfully affect the outcomes in a variety of voting settings.

Considerable attention has been devoted to the incentives of agents to vote truthfully in the literature on Social Choice. There are numerous studies characterizing social choice functions that satisfy strategy-proofness (i.e., truth-telling is a weakly dominant strategy in the direct mechanism). Arguably, there are social choice functions satisfying desirable properties that fail to be strategyproof. If we insist on the desirability of those social choice functions, we should measure their manipulability. Although we lack a unanimously way of doing so theoretically, laboratory experiments provide an excellent way of addressing this question empirically; in fact, survey data can lead to errors in estimating voting behavior (Wright, 1990; Alvarez and Nagler, 2000). This paper is part of a more ambitious project in which we want to test experimentally several well-known social choice functions in their appropriate domains of definition. The current paper can be understood as a first step in this direction.

\section{Acknowledgements}

We would like to thank two anonymous referees for their valuable feedback that really helped to shape the exposition of the paper. We also benefited from comments and suggestions provided by seminar and conferences participants at the Social Choice and Mechanism Design Workshop in Manchester, Middlesex University London, University of Hamburg (UHH). Special thanks to Aniol Llorente-Saguer, Matthias Weber, Paul J. Ferraro, Leeat Yariv, Charlie Plott, Rebecca B. Morton, Daniel Müller, Moti Michaeli, Gary Bolton, Ben Greiner, Collin Raymond, David Hugh-Jones, and Jonathan Lafky who provided very useful references and stimulated discussion through the ESA Group. Financial support from Junta de Andalucía (SEJ-5980) and the Spanish Ministry of Science and Technology (ECO2014-53767-P and ECO2014-58297-R) is gratefully acknowledged. Ismael Rodriguez-Lara wants to thank financial support from FEDER and the Ministerio de 
Economia y Competitividad under the project CO2017-87245-R. 


\section{References}

[1] Abeler, J., Nosenzo, D., \& Raymond, C. (2016). Preferences for truth-telling: A meta study. Econometrica, forthcoming.

[2] Agranov, M., Goeree, J. K., Romero, J., \& Yariv, L. (2017). What makes voters turn out: The effects of polls and beliefs. Journal of the European Economic Association, forthcoming.

[3] Ai, C., \& Norton, E. C. (2003). Interaction terms in logit and probit models. Economics Letters, 80(1), 123-129.

[4] Alvarez, R. M., \& Nagler, J. (2000). A new approach for modelling strategic voting in multiparty elections. British Journal of Political Science, 30(1), 57-75.

[5] Anderson, L.R., C.A. Holt, K.K. Sieberg and A.L. Oldham (2015). An Experimental Study of Jury Voting Behavior, in N. Schofield, G. Caballero (eds.), The Political Economy of Governance: Institutions, Political Performance and Elections. Berlin: Springer, 157-178.

[6] Anderson, L. R., \& Holt, C. A. (1997). Information cascades in the laboratory. The American Economic Review 87(5), 847-862.

[7] Azrieli, Y., Chambers, C. P., \& Healy, P. J. (2018). Incentives in experiments: A theoretical analysis. Journal of Political Economy, forthcoming.

[8] Battaglini, M., Morton, R. B., \& Palfrey, T. R. (2010). The swing voter's curse in the laboratory. The Review of Economic Studies, 77(1), 61-89.

[9] Barberà , S. \& Nicolò, A. (2016). Information Disclosure Under Strategy-Proof Voting Rules, mimeo.

[10] Bartling, B., Fischbacher, U., \& Schudy, S. (2015). Pivotality and responsibility attribution in sequential voting. Journal of Public Economics, 128, 133-139.

[11] Bassi, A. (2015). Voting systems and strategic manipulation: An experimental study. Journal of Theoretical Politics, 27(1), 58-85.

[12] Bassi, A., Morton, R. B., \& Williams, K. C. (2011). The effects of identities, incentives, and information on voting. The Journal of Politics, 73(02), 558-571. 
[13] Blais, A. \& Hortala-Vallve, R. (2016). Are people more or less inclined to vote when aggregate turnout is high? In: Voting Experiments, Blais, A., Laslier, J. F, \& Van der Straeten, K. (ed), pp. $117-126$

[14] Blais, A. \& Hortala-Vallve, R. (2017). Conformity and turnout, mimeo.

[15] Bouton, L., Castanheira, M., \& Llorente-Saguer, A. (2017a). Multicandidate elections: Aggregate uncertainty in the laboratory. Games and Economic Behavior, 101, 132-150.

[16] Bouton, L., Llorente-Saguer, A., \& Malherbe, F. (2017b). Unanimous rules in the laboratory. Games and Economic Behavior, 102, 179-198.

[17] Brandts, J., \& Charness, G. (2011). The strategy versus the direct-response method: a first survey of experimental comparisons. Experimental Economics, 14(3), 375-398.

[18] Callander, S. (2007). Bandwagons and momentum in sequential voting. The Review of Economic Studies, 74(3), 653-684.

[19] Coate, S., \& Conlin, M. (2004). A group rule: Utilitarian approach to voter turnout: Theory and evidence. American Economic Review, 1476-1504.

[20] Corazzini, L., \& Greiner, B. (2007). Herding, social preferences and (non-) conformity. Economics Letters, 97(1), 74-80.

[21] Dorff, R. H., \& Brenner, S. (1992). Conformity Voting on the United States Supreme Court. The Journal of Politics, 54(03), 762-775.

[22] Duffy, J., \& Tavits, M. (2008). Beliefs and voting decisions: A test of the pivotal voter model. American Journal of Political Science, 52(3), 603-618.

[23] Dutta, B., \& Sen, A. (2012). Nash implementation with partially honest ividuals. Games and Economic Behavior, 74(1), 154-169.

[24] Eguia, J. X. (2011). Voting blocs, party discipline and party formation. Games and Economic Behavior, 73(1), 111-135.

[25] Esponda, I., \& Vespa, E. (2014). Hypothetical thinking and information extraction in the laboratory. American Economic Journal: Microeconomics, 6(4), 180-202. 
[26] Feddersen, T., Gailmard, S., \& Sandroni, A. (2009). Moral bias in large elections: Theory and experimental evidence. American Political Science Review, 103(02), 175-192.

[27] Feddersen, T., \& Pesendorfer, W. (1998). Convicting the innocent: The inferiority of unanimous jury verdicts under strategic voting. American Political Science Review, 92(01), 23-35.

[28] Feddersen, T., \& Sandroni, A. (2006). A theory of participation in elections. The American Economic Review, 96(4), 1271-1282.

[29] Feltovich, N. (2003). Nonparametric tests of differences in medians: comparison of the Wilcoxon- Mann-Whitney and robust rank-order tests. Experimental Economics, 6(3), 273297.

[30] Fischbacher, U. (2007). z-Tree: Zurich toolbox for ready-made economic experiments. Experimental Economics, 10(2), 171-178.

[31] Gerber, E. R., Morton, R. B., \& Rietz, T. A. (1998). Minority representation in multimember districts. American Political Science Review, 92(01), 127-144.

[32] Goeree, J. K., \& Yariv, L. (2015). Conformity in the Lab. Journal of the Economic Science Association, 1-14.

[33] Gerber, E., Morton, R. \& Rietz, T. (1998). ?Minority Representation in Multimember Districts. American Political Science Review, 92(03), 127-144.

[34] Guarnaschelli, S., McKelvey, R. D., \& Palfrey, T. R. (2000). An experimental study of jury decision rules. American Political Science Review, 94(02), 407-423.

[35] Höchtl, W., Sausgruber, R., \& Tyran, J. R. (2012). Inequality aversion and voting on redistribution. European Economic Review, 56(7), 1406-1421.

[36] Hung, A. A., \& Plott, C. R. (2001). Information cascades: Replication and an extension to majority rule and conformity-rewarding institutions. American Economic Review, 1508-1520.

[37] Karaca-Mandic, P., Norton, E. C., \& Dowd, B. (2012). Interaction terms in nonlinear models. Health Services Research, 47(1), 255-274.

[38] Kube, S., \& Puppe, C. (2009). (When and how) do voters try to manipulate?. Public Choice, $139,39-52$. 
[39] Levine, D., \& Palfrey, T. (2007). The paradox of voter participation? A laboratory study. American Political Science Review, 101, 143?58.

[40] Lundquist, T., Ellingsen, T., Gribbe, E., \& Johannesson, M. (2009). The aversion to lying. Journal of Economic Behavior and Organization, 70(1), 81-92.

[41] Luzzati, T. (1999). Economic theory and conformism (Vol. 68). Edward Elgar Publishing, Cheltenham Glos, UK.

[42] Michaeli, N., \& Spiro, D., (2015). Norm Conformity across Societies. Journal of Public Economics, 132, 51-65.

[43] Morelli, M. (2004). Party formation and policy outcomes under different electoral systems. Review of Economic Studies 71(3), 829-853.

[44] Moreno, B. \& Ramos-Sosa, M.P. (2017) Conformity in voting. Social Choice and Welfare, 48: 519-543.

[45] Morton, R. B., Muller, D., Page, L., \& Torgler, B. (2015). Exit polls, turnout, and bandwagon voting: Evidence from a natural experiment. European Economic Review, 77, 65-81.

[46] Morton, R. B., \& Ou, K. (2015). What motivates bandwagon voting behavior: Altruism or a desire to win?. European Journal of Political Economy, 40, 224-241.

[47] Morton, R. B., Piovesan, M., \& Tyran, J. R. (2012). The dark side of the vote: Biased voters, social information, and information aggregation through majority voting. Harvard Business School Research Paper, (13-017).

[48] Morton, R. B., \& Williams, K. C. (1999). Information asymmetries and simultaneous versus sequential voting. American Political Science Review, 93(01), 51-67.

[49] Morton, R. B., \& Williams, K. C. (2000). Learning by voting: Sequential choices in presidential primaries and other elections. University of Michigan Press.

[50] Palfrey, T. R. (2016). Experiments in Political Economy. In: The Handbook of Experimental Economics, Kagel, J., \& Roth, A. (ed), pp. 347?434. Princeton University Press.

[51] Rivas, J., \& Rodriguez-Alvarez, C. (2017). Deliberation, Leadership and Information Aggregation. The Manchester School, 85(4), 395-429. 
[52] Sanchez-Pages, S., \& Vorsatz, M. (2007). An experimental study of truth-telling in a senderreceiver game. Games and Economic Behavior, 61(1), 86-112.

[53] Selten, R. (1967). Die Strategiemethode zur Erforschung des eingeschränkt rationalen Verhaltens im Rahmen eines Oligopolexperiments. In H. Sauermann (Ed.), Beiträge zur experimentellen Wirtschaftsforschung (pp. 136-168). Tübingen: Mohr.

[54] Smith, V. L. (1976). Experimental economics: Induced value theory. The American Economic Review, 66(2), 274-279.

[55] Tyran, J. R. (2004). Voting when money and morals conflict: an experimental test of expressive voting. Journal of Public Economics, 88(7), 1645-1664.

[56] Tyran, J. R., \& Sausgruber, R. (2006). A little fairness may induce a lot of redistribution in democracy. European Economic Review, 50(2), 469-485.

[57] Van der Straeten, K., Laslier, J. F., Sauger, N., \& Blais, A. (2010). Strategic, sincere, and heuristic voting under four election rules: an experimental study. Social Choice and Welfare, $35(3), 435-472$.

[58] Van der Straeten, K., Sauger, N., Laslier, J. F., \& Blais, A. (2013). Sorting out mechanical and psychological effects in candidate elections: An appraisal with experimental data. British Journal of Political Science, 43(4), 937-944.

[59] Van der Straeten, K., Laslier, J. F., \& Blais, A. (2016). Patterns of Strategic Voting in Run-Off Elections. In: Voting Experiments, Blais, A., Laslier, J. F, \& Van der Straeten, K. (ed), pp. 215-236.

[60] Wright, G. C. (1990). Misreports of vote choice in the 1988 NES senate election study. Legislative Studies Quarterly, 15(4), 543-563. 


\section{PREVIOUS WORKING PAPERS IN THE SERIES}

On how to allocate the fixed cost of transport networks.

Teresa Estañ, Natividad Llorca, Ricardo Martínez, and Joaquín Sánchez-Soriano

Multilevel proficiency comparisons with an application to educational outcomes in PISA.

Ricardo Martínez and Antonio Villar

Population structure and the human development index.

Carmen Herrero, Ricardo Martínez, and Antonio Villar

From Financial to Managerial Capitalism. The slow adaptation of Spanish corporate elite Downloads.

Juan A. Rubio Mondéjar, Jósean Garrués Irurzun and Luis Chirosa

Sequential Common Consequence Effect and Incentives Downloads.

María J. Ruiz-Martos

The Fiscal and Welfare Consequences of the Price Indexation of Spanish Pensions.

Julian Díaz Saavedra

Allocating the costs of cleaning a river; estimating responsibilities versus incentive compatibility.

Jorge Alcalde-Unzu, María Gómez-Rua and Elena Molis

Changes in Subjective Well-Being Over Time in Germnay.

Ana I. Moro Egido, María Navarro and Ángeles Sánchez-Domínguez

Catalonia; Independence and Pensions.

Javier Díaz Giménez and Javier Díaz Jiménez

Intra-household allocation of resources and decision-making. How important are in terms of individual well-being?.

Elena Bárcena-Martín, Maite Blázquez and Ana I. Moro Egido

Random Lottery Incentive Mechanism in Dynamic Choice Experiments.

María J. Ruiz Martos 